\title{
Equipment and methods for synthetic aperture anatomic and flow imaging
}

\author{
Jensen, Jørgen Arendt; Nikolov, Svetoslav; Misaridis, Thanassis; Gammelmark, Kim
}

Published in:

IEEE Ultrasonics Symposium, 2002. Proceedings

Link to article, DOI:

10.1109/ULTSYM.2002.1192591

Publication date:

2002

Document Version

Publisher's PDF, also known as Version of record

Link back to DTU Orbit

Citation (APA):

Jensen, J. A., Nikolov, S., Misaridis, T., \& Gammelmark, K. (2002). Equipment and methods for synthetic aperture anatomic and flow imaging. In IEEE Ultrasonics Symposium, 2002. Proceedings (pp. 1518-1527). IEEE. https://doi.org/10.1109/ULTSYM.2002.1192591

\section{General rights}

Copyright and moral rights for the publications made accessible in the public portal are retained by the authors and/or other copyright owners and it is a condition of accessing publications that users recognise and abide by the legal requirements associated with these rights.

- Users may download and print one copy of any publication from the public portal for the purpose of private study or research.

- You may not further distribute the material or use it for any profit-making activity or commercial gain

- You may freely distribute the URL identifying the publication in the public portal 


\title{
Equipment and methods for synthetic aperture anatomic and flow imaging
}

\author{
Jørgen Arendt Jensen, Svetoslav I. Nikolov, Thanassis Misaridis and Kim L. Gammelmark \\ Center for Fast Ultrasound Imaging, Ørsted•DTU, Bldg. 348, \\ Technical University of Denmark, DK-2800 Kgs. Lyngby, Denmark
}

\begin{abstract}
Conventional ultrasound imaging is done by sequentially probing in each image direction. The frame rate is, thus, limited by the speed of sound and the number of lines necessary to form an image. This is especially limiting in flow imaging, since multiple lines are used for flow estimation. Another problem is that each receiving transducer element must be connected to a receiver, which makes the expansion of the number of receive channels expensive.
\end{abstract}

Synthetic aperture (SA) imaging is a radical change from the sequential image formation. Here ultrasound is emitted in all directions and the image is formed in all directions simultaneously over a number of acquisitions. SA images can therefore be perfectly focused in both transmit and receive for all depths, thus significantly improving image quality. A further advantage is that very fast imaging can be done, since only a few emissions are needed for forming an image, and a novel approach of recursive ultrasound imaging can be used to give several thousand images a second. A commercial SA imaging system has, however, not yet been introduced due to a number of problems. The fundamental problems are primarily that the signal-to-noise ratio and penetration depth are low and velocity imaging is thought not to be possible.

This paper will address all the issues above and show that they can all be solved using various techniques. The SNR is increased significantly beyond that for normal systems by using coded imaging and grouping of elements to form larger defocused emitting apertures. It is also possible to have many more receive channels, since different elements can be sampled during different emissions. The paper also shows that velocity imaging can be performed by making a special grouping of the received signals without motion compensation by using recursive imaging. With this technique continuous imaging at all points in the image is possible, which can significantly improve velocity estimates, since the estimates can be formed from a large number of emissions (100-200). The research scanner RASMUS, capable of acquiring clinical SA images, has been constructed and will be described. A number of phantom and in-vivo images will be presented showing in-vivo SA B-mode and flow imaging.

\section{INTRODUCTION}

Modern digital ultrasound scanners acquire all images sequentially, where one direction in the image is measured at a time. The consequence of this is that the frame rate is limited by the image size and the speed of sound. The frame rate can be expressed as:

$$
f_{r}=\frac{c}{2 D N_{l}},
$$

where $c$ is the speed of sound, D is the depth and $N_{l}$ is the number of lines in one image. For normal anatomic imaging, this is in general not a problem. For flow imaging problems are encountered, since 8 or 16 emissions are necessary for each direction, and the frame rate is correspondingly reduced. Imaging the heart down to $15 \mathrm{~cm}$ and creating 100 image lines gives an unsatisfactory frame rate of 6.4 or 3.2 $\mathrm{Hz}$ (16 emissions/direction). This has been attempted solved by using explososcan [1], where a slightly broader beam is emitted with $e$.g. four lines being simultaneously created in receive to yield a four times increase in frame rate. The drawback is the slightly wider beam, where the effects of the single transmit focus is evident.

Another problem in conventional imaging is the single transmit focus, where the image is only narrowly focused at a single depth. This is often compensated for by making compound imaging, where one image line is made from several emissions with different transmit foci. The drawback here is the degradation in frame rate.

The frame rate problem also influences color flow mapping, since the number of emissions for one direction has to be kept to a minimum. The standard deviation of the estimates are therefore high. Another problem is that only the flow along the ultrasound beam is found, and a transverse flow will no be shown. The correct and accurate velocity is, thus, not shown in the current systems.

All of the problems are amplified when going to real-time three-dimensional scanning. To maintain a good frame rate, 16 beams must be reconstructed for each emission. This can give 15 volumes per second in the Duke 3D system [2], but is 


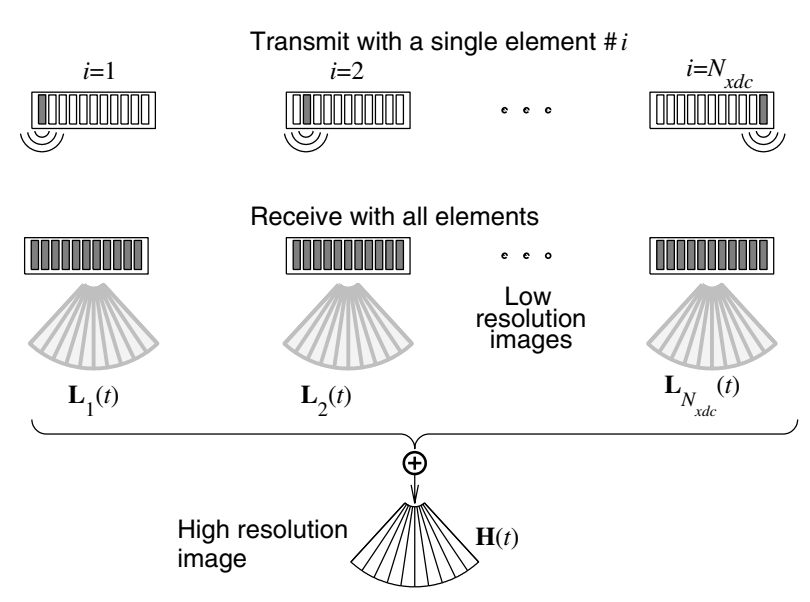

Figure 1: Principle of synthetic aperture imaging.

not sufficient to yield 3D flow data in real time. The system also have to use a sparse 2D array and have many channels in order to yield a good image quality.

The current systems, thus, have many limitations, which all can be related to the sequential data acquisition, that is limited by the speed of sound in tissue.

A natural step is to devise methods where parallelism is employed, and where the image can be made from only a few emissions. Many authors have investigated synthetic aperture imaging as a possible solution $[3,4,5,6,7,8]$. There are, however, also problems here. The penetration depth is limited, since the emitted field is spread out in all directions. Flow imaging is also thought not to be possible, since the image is constructed from a number of emissions. Signals from moving scatterers will therefore not be in phase, and velocity estimation is therefore believed to be impossible.

This paper will give a brief introduction to synthetic aperture imaging, and it will be shown that the problems with limited penetration depth and flow estimation can be solved. Equipment constructed for measuring such images will be described and examples of in-vivo anatomic and flow synthetic aperture images are presented.

\section{SynthetiC APERTURE IMAGING}

The principle of synthetic aperture (SA) imaging is shown in Fig. 1. A defocused wave is emitted from a single element and the signal is received on all elements of the transducer. The geometric distance from the emission to the imaging point and back can then be calculated, and a low resolution image can be formed for all points in the image. The process is then repeated for other emitting elements and a series of low resolution images are formed. All of these are focused for the given transmission and receiving elements, and summing them gives a high resolution image, that is focused for all transmissions and receptions corresponding to a dynami-

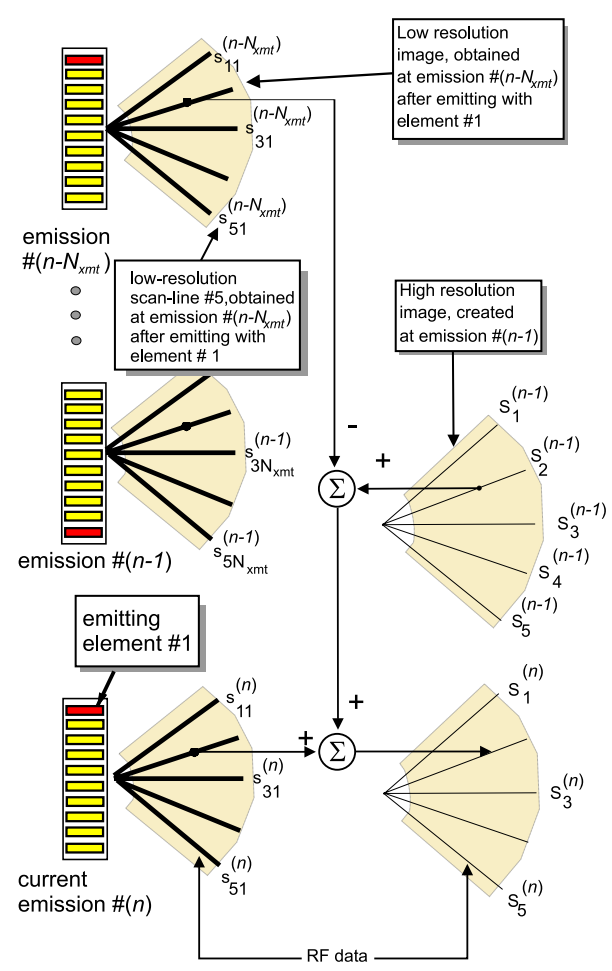

Figure 2: Recursive ultrasound imaging. In transmit only one element is excited. Multiple receive beams are formed simultaneously for each transmit pulse. Each element is excited again after $N_{x m t}$ emissions where $N_{x m t}=N_{x d c}=10$ in this example (from [9]).

cally focused image in both transmit and receive.

This type of synthetic aperture imaging, thus, gives the best possible resolution for delay-sum beamforming at all places in the image. The number of receiving elements can also change per emission, and many more elements than in traditional imaging can be employed with less electronics. Also the frame rate can be increased, since a full image can be constructed for only a few emissions.

SA imaging can also be used for recursive ultrasound imaging [9], where an image is created after each pulse emission. This is possible since a high resolution image can be made at all times after a complete set of low resolution images have been acquired. It is, thus, possible to take the last $N_{x m t}$ emissions to form an image, where $N_{x m t}$ is the number of emissions for one high resolution image. It is sufficient to replace the oldest low resolution image with the last as shown in Fig. 2, if the data acquisition is performed continuously. A new image can then be made after each pulse emission, and the frame rate can be maintained at the pulse repetition frequency.

The drawbacks of SA imaging are that the image is formed over several emissions, so it is prone to tissue motion artifacts, and it cannot be used for flow imaging. Also only one 


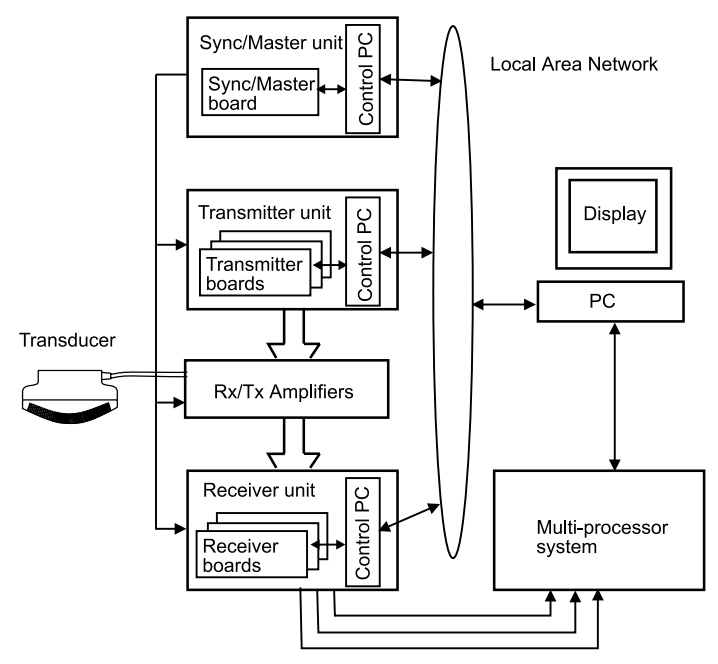

Figure 3: Overall diagram of system (from [10]).

element is used for a defocused transmission, and very little energy is therefore send into the tissue resulting in a low penetration depth. Sections IV and V will show that the penetration problem can be solved, and that SA imaging can attain a better penetration depth than traditional imaging. The clinical images in Section $\mathrm{V}$ also shows that motion is a minor problem for a typical clinical scanning. Section VII will show that it can be used for flow imaging with better results than traditional color flow map imaging. The final problem is how to construct a SA imaging system and this is addressed in the next Section.

\section{EQUIPMENT FOR SYNTHETIC APERTURE IMAGING}

One of the main problems for investigating SA imaging has been that there does not exist systems for acquiring and processing the necessary data in real-time. We therefore set out in 1997 to build a fully flexible system, that could be used for all types of imaging. The system should have enough memory to sample RF data for each individual transducer element over a couple of seconds, and the transmitters should be capable of sending any kind of signal for any element and emission number. A full description of the system can be found in [10].

The RASMUS (Remotely Accessible Software configurable Multi-channel Ultrasound Sampling) system consists of four distinct modules: The transmitters, the analog amplifiers $(\mathrm{Rx} / \mathrm{Tx}$ amplifiers), receivers, and the sync/master unit. The main blocks are depicted in Fig. 3.

Two 19 inch racks houses the 8 transmitters and the 8 receivers, respectively, as shown in Fig. 4. Each of these racks also houses a slot PC running Linux, which controls the setup and operation of the boards. A separate enclosure is used for

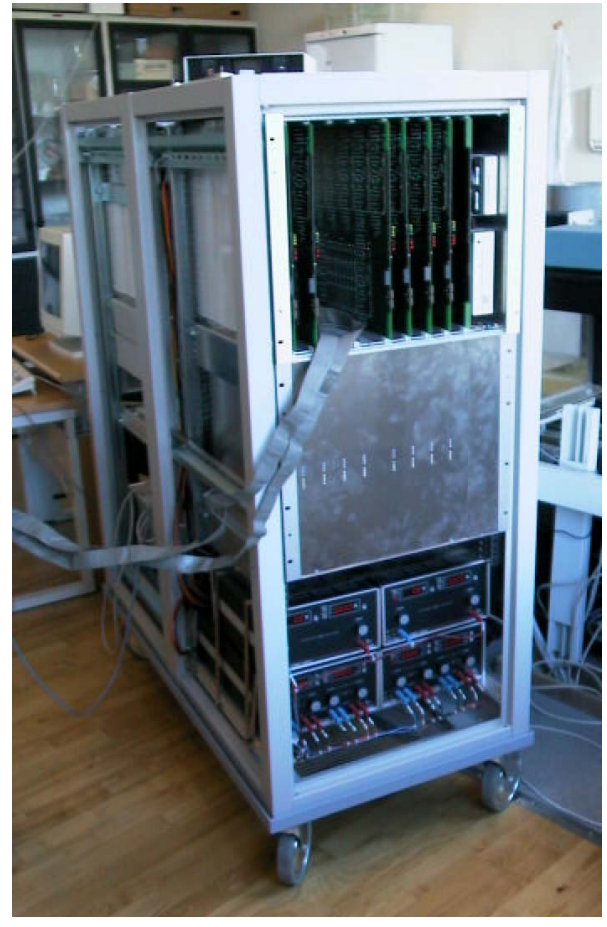

Figure 4: The RASMUS scanner seen from the front with the receivers on the top, transmitters in the middle and the power supplies in the bottom.

the analog front-end, which is shielded from the digital electronics. Here linear laboratory power supplies are also used to supply the front-end to keep the noise low.

\section{Transmitter}

Each transmitter board has 16 channels, which consists of a 128 ksamples RAM connected to a $40 \mathrm{MHz}, 12$ bits DAC. The RAM is controlled by an FPGA (Field Programmable Gate Array), where the individual waveforms are selected as a memory start address and a transmit delay. Both the waveform and the delay can change for each emission, which ensures full flexibility in the transmissions. The length of the waveforms can be set, and waveform durations up to $100 \mu \mathrm{s}$ can be emitted.

The system houses 8 boards for a total of 128 independent emission channels.

\section{Receiver}

The Receiver board is illustrated in Fig. 5. It samples and processes 8 analog signals selected from 16 inputs through a 2-to-1 multiplexer. Each of the input signals are sampled at $40 \mathrm{MHz}$ and 12 bits into one of the two SRAMs. The one SRAM is used for sampling and the second is used for transferring data to the Focus FPGA associated with each channel. The data is then processed in the Focus FPGA using param- 


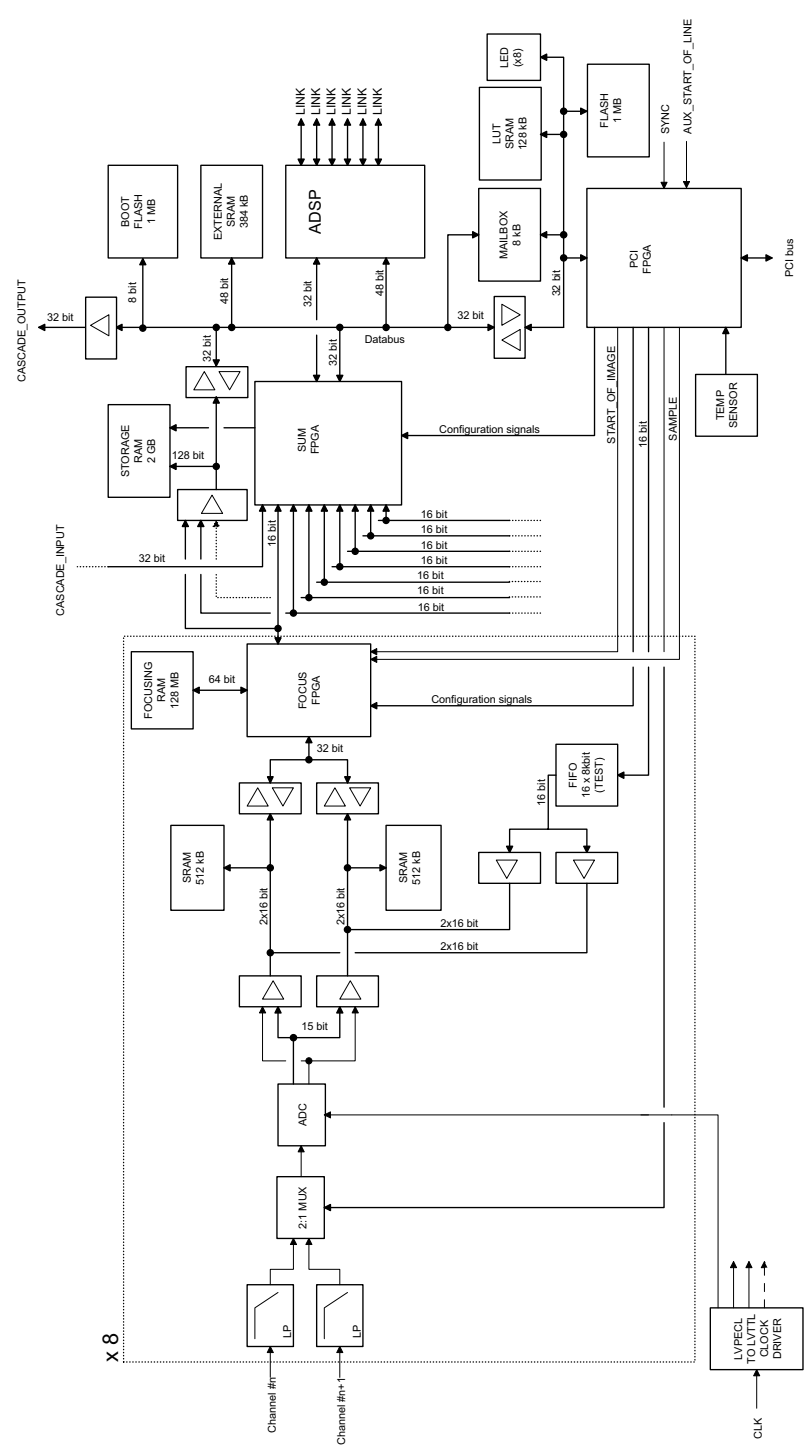

Figure 5: Main diagram of Receiver board (from [10]).

eters from the 128 MBytes Focusing RAM, and the result is passed on to the Sum FPGA. The processing can either be a dynamic receive focusing or the data can be passed unaltered for later storage. The Sum FPGA can either store the data in the 2Gbytes storage RAM or it can sum all 8 channels with the result from the cascade bus and pass it on to the next receiver board through the cascade bus. The last receiver board in the system then transmits the focused signal to the ADSP SHARC for transmission to the display PC.

The storage RAM can contain more than 3 seconds of real time data for each channel, that later can be accessed from the PCs controlling the system.

The actual board layout is shown in Fig. 6. All boards in the system has a size of $53 \mathrm{~cm}$ by $36.5 \mathrm{~cm}$, and the receiver is manufactured using 12 layers.

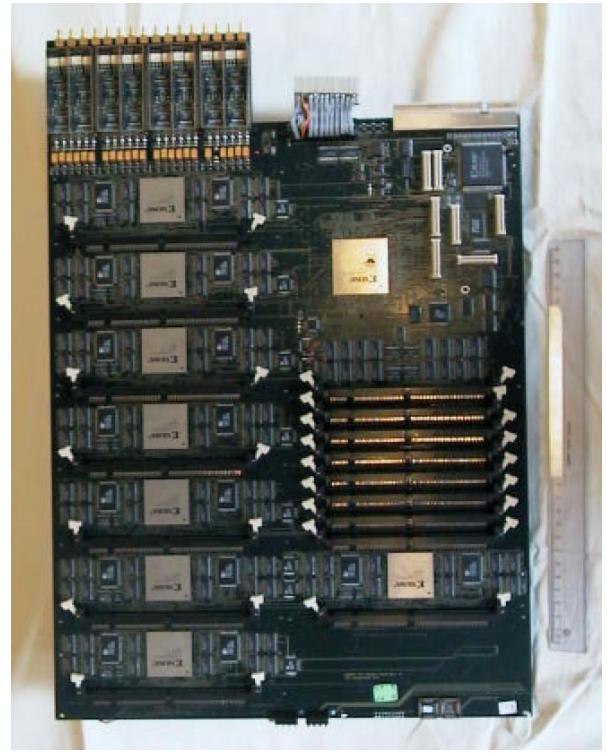

Figure 6: The receiver board layout without RAM modules to reveal the processing electronics. The board size is $53 \mathrm{~cm}$ by $36.5 \mathrm{~cm}$.

\section{Software control}

From the software point of view, the system consists of several computers that are directly connected to a number of transmitter and receiver boards. The computers are linked by a 100 Mbit LAN, and uses Linux as operating system and $\mathrm{TCP} / \mathrm{IP}$ as the underlying communication protocol. A server program is running on each of the slot PCs and client programs can connect to the servers and setup the system.

The interface to the client program is implemented as a MATLAB toolbox and can be run by any PC connected to the net. The function calls are implemented to be as close to the functions in the simulation program Field II [11] as possible. Algorithms created using Field II can hereby easily be tested on the scanner with only minor changes in the Matlab program.

An example for setting the system to perform phased array B-mode imaging is shown below:

$\%$ Auto-detect and initialize the system sys_init('auto');

$\%$ Set the pulse repetition frequency sys_set_fprf( $($ prf $f)$;

$\%$ Set the sampling range gate in receive sys_set_sampling_interval(start_depth, end_depth);

$\%$ Set the number of scan-lines per frame sys_set_no_lines(no_lines); $\%$ Define the transducer. Necessary for the delay calculations tr_linear_array(no_elements, width, height, kerf);

$\%$ Do for all lines in the image:

for line no = 1:no_lines 
$\%$ Set the pulse and the apodization for the current line

xmt_excitation(wave form (line_no));

xmt_apodization(line_no,xmt_apo(line_no,: ));

rcv_apodization(line_no,times, rcv_apo(line_no,:,: ));

$\%$ Set the focus, defined in 3D coordinates

xmt_focus(line_no,focus(line_no));

rcv_dynamic_focus(line_no, theta(line_no), fi(line_no)); end

$\%$ Set the time-gain compensation curve

tmg_tgc(tgc_vector);

$\%$ Start the continuous imaging process tmg_start

In order to make the system perform linear array imaging only one line needs to be added, which changes the origin of the individual scan-lines.

\section{SOLVING THE PENETRATION PROBLEM}

One of the main problems with SA is that only a single element is used for the emission. The spherical wave emitted will spread out in all directions, and the emitted intensity is therefore low, severely limiting the depth of penetration.

One approach to increase the energy is to use a number of elements to synthesize the emitted spherical wave thereby increasing the emitted energy. As showed experimentally by Karaman and coworkers [12], the amplitude of the wavefront created by the defocused subaperture consisting of $N_{t}$ elements is proportional to $\sqrt{N_{t}}$. Using 11 elements, thus, gives an increase of $10.4 \mathrm{~dB}$.

A second method is to use a coded excitation to increase the transmitted energy. Using a frequency modulated chirp and a matched filter for compression can increase the signalto-noise ratio by a factor $T_{c} B /\left(T_{p} B_{p}\right)$, where $T_{c}$ is the duration of the chirp and $B$ is the bandwidth, and $T_{p}$ and $B_{p}$ are for the corresponding pulse excitation. An increase of 10 to $15 \mathrm{~dB}$ can be attained without a reduction in axial resolution and contrast $[13,14]$.

Combining the two approaches can give a significant increase in SNR, when it is also taken into account that the SA image is made from combining all the measurements [15]. The improvement in signal-to-noise ratio compared to an ordinary linear array image is [16]:

$$
I_{B}=\frac{M N_{t}}{N_{e}^{2}} \frac{T_{c}}{T_{p}},
$$

where $M$ is the number of SA emissions and $N_{e}$ is the number of emitting elements for linear array scanning. For the typical case of a $7 \mathrm{MHz}$ system with $M=96, N_{e}=64, N_{t}=33, T_{c}=$ $20 \mu \mathrm{s}, T_{p}=0.29 \mu \mathrm{s}$, and 128 receiving elements, the scheme gives an improvement factor $I_{B}$ of $17 \mathrm{~dB}$ indicating that the SA system has a larger depth of penetration.

\section{ANATOMIC SYNTHETIC APERTURE IMAGES}

An example of images measured by the RASMUS system using the method described in the previous Section is shown in Fig. 7, where a number of nylon wires embedded in a tissue mimicking phantom with an attenuation of $0.5 \mathrm{~dB} /[\mathrm{MHz}$ $\mathrm{cm}$ ] has been scanned. A 128 element linear $7 \mathrm{MHz}$ array probe has been used with the system described in Section III. The left image shows the traditional linear array scan and the right image shows the SA image using 33 elements for each transmission together with a $20 \mu$ s chirp. It can be seen that the wires are better defined and that the penetration depth has been increased by roughly $40 \%$ from $70 \mathrm{~mm}$ to $100 \mathrm{~mm}$.

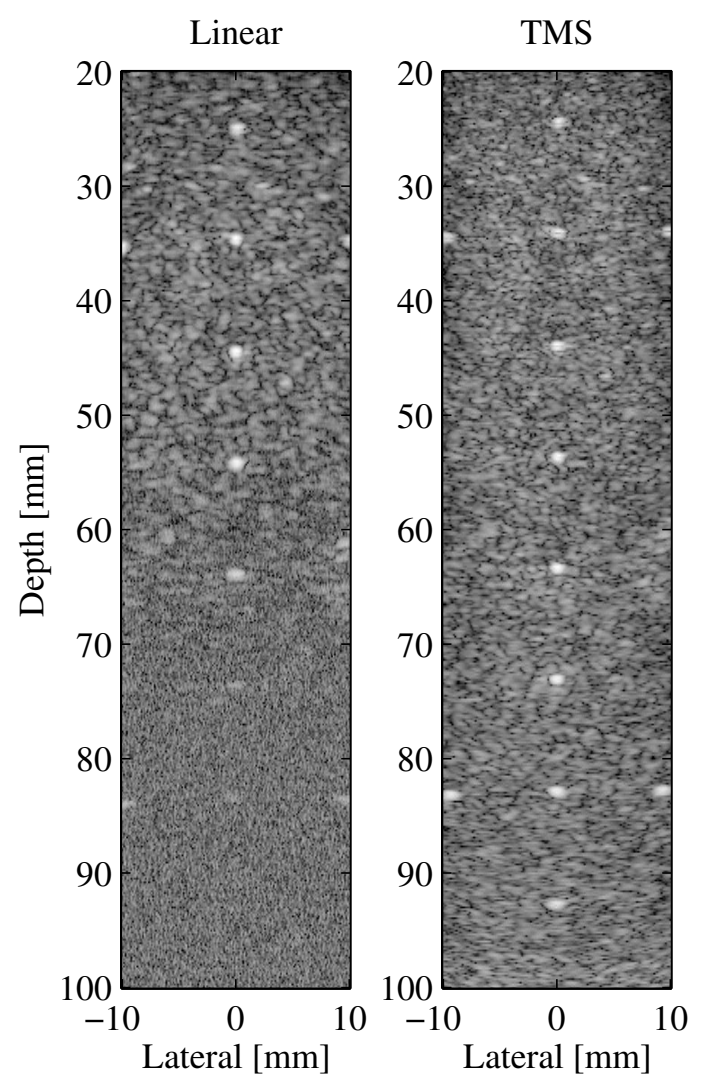

Figure 7: Linear array and synthetic aperture scan of nylon wires in a tissue mimicking phantom with an attenuation of $0.5 \mathrm{~dB} /[\mathrm{MHz} \mathrm{cm}]$ (from [16]).

Figure 8 shows in-vivo images of the abdomen of a healthy 27 year old male for conventional imaging (left) and synthetic imaging (right) using a convex array transducer. The transducer used for the measurement is a commercial $5.5 \mathrm{MHz}$ convex array transducer with 192 elements and $\lambda$ pitch.

For conventional imaging a 64 elements, Hamming weighted, transmit aperture is used with a fixed focus at 70 $\mathrm{mm}$. A 2 cycle sinusoid at $5.5 \mathrm{MHz}$ weighted with a Hanning window is used as the excitation pulse, and all 128 el- 

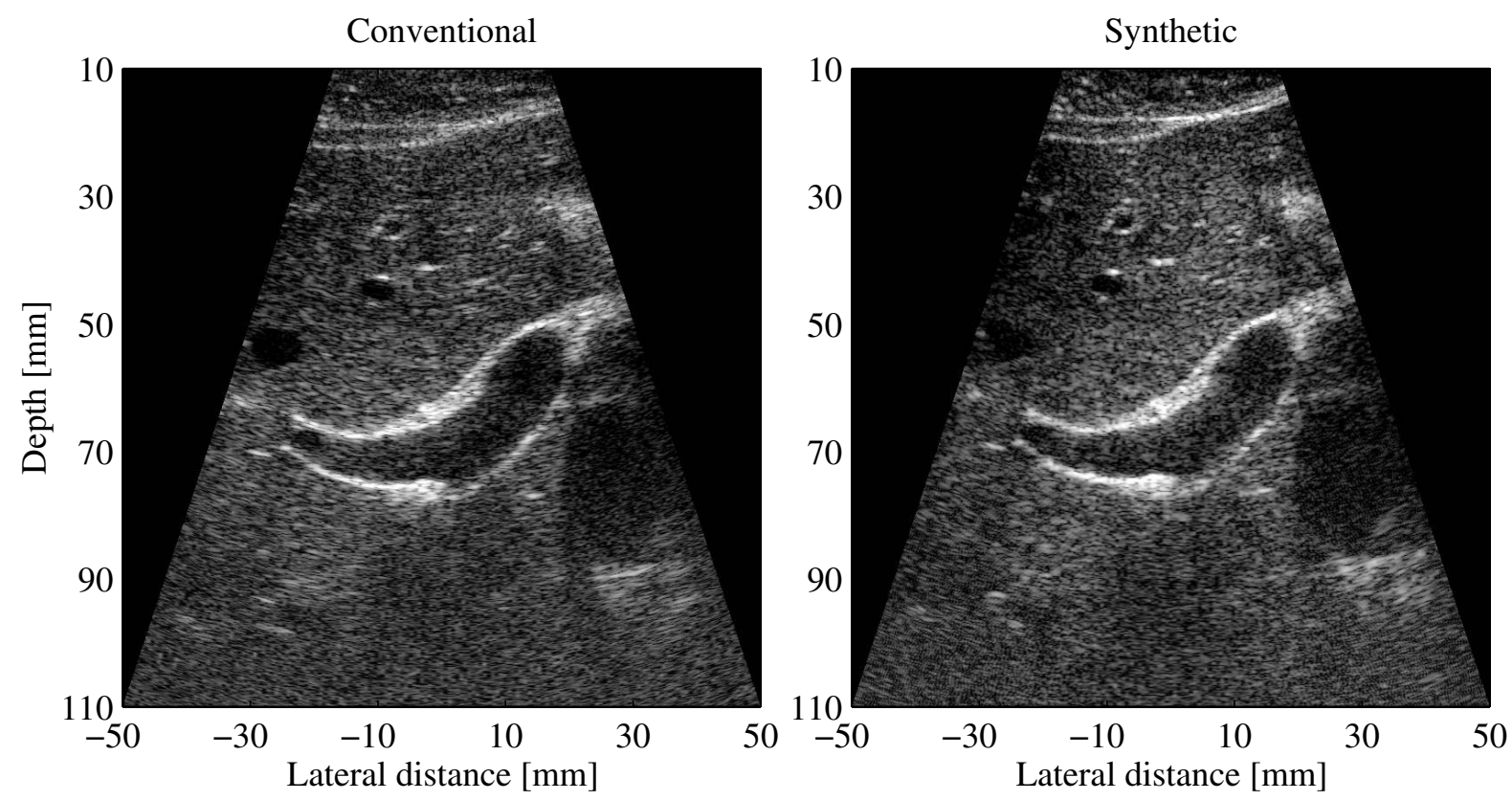

Figure 8: In-vivo images of the abdomen of a healthy 27 years old male using a convex array transducer. The left is the conventional convex array image and the right is the SA image.

ements are used on receive and for the subsequent beamformation. For synthetic imaging an 11 element subaperture is used to emulate the spherical wave transmission, and all 128 elements in the aperture are used on receive. The center of the transmit aperture is moved one element after each transmission, which results in a total of 118 emissions. A $20 \mu$ s linear FM signal with a center frequency of $5.5 \mathrm{MHz}$ and $90 \%$ bandwidth is used as excitation signal. The FM signal has been weighted with a Tukey window to reduce the temporal sidelobes [13].

The conventional data are beamformed using dynamic receive focusing with delay updates for each sample. When beamforming both sets of data the element sensitivity is taken into account. The acceptance angle [6] is set to \pm 50 degrees for each element, and, thus, an element is not applied in the beamformer until the point to be beamformed lies inside its acceptance angle.

Figure 8 shows a region of the abdomen, where there is motion from the heart and the pulsation of the vessels. It can been seen that the SA image is still of high quality despite the motion, and motion artifacts, thus, plays a minor role in SA imaging for this case.

\section{REAL-TIME THREE-DIMENSIONAL IMAGING}

SA imaging can also be used for creating three-dimensional images in real time. One approach is to use a two-

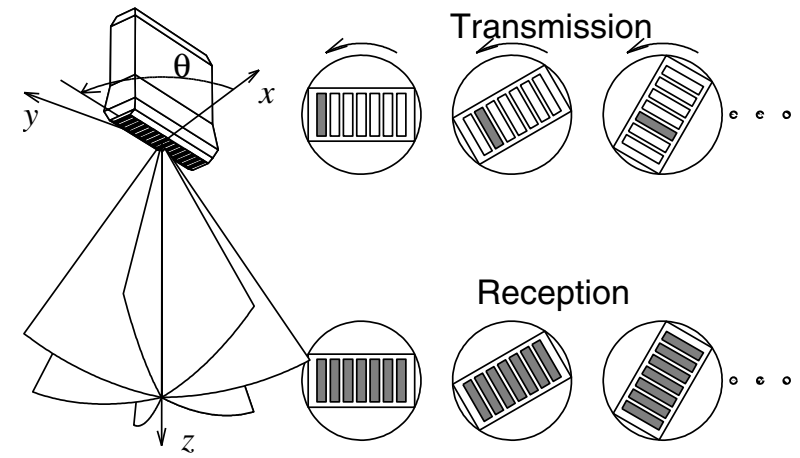

Figure 9: Three-dimensional scanning geometry.

dimensional array and then multiplex between the elements to measure from a large number of elements to have a good image quality [17]. Another approach is to use a rotating phased array transducer for sampling all spatial locations in a rotational movement. Such an approach is shown in Fig. 9. Here the array is continuously rotated and the synthetic aperture data are acquired from different positions. The position of the different receiving elements will be different for the different emissions, but this can be handled in the beamformation process by keeping track of the transducer position.

For the given position a number of low resolution images will partly overlap as shown in Fig. 10. These can be used in the beamformation process to form the high resolution images. The overlap will be high at the center and less at the 


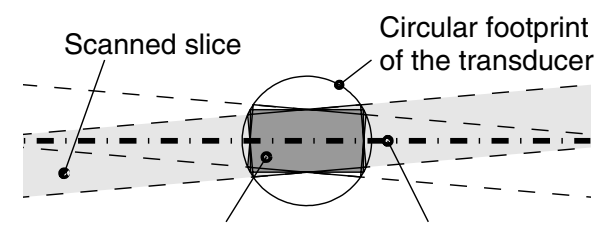

The array

Beamformed plane

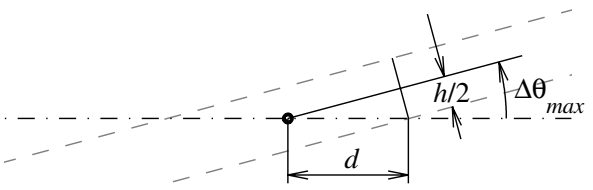

Figure 10: Slice reconstruction.

edges, so that less low resolution images can be used at the edge of the images.

Such a system has been implemented on the RASMUS system together with a rotating phased array transducer from Vermon [18]. The $3 \mathrm{MHz}$ phased array transducer has 64 elements and can rotate back and forth more than 10 times per second to generate 10 volumes a second. Using an $f_{p r f}$ of $5 \mathrm{kHz}$ gives 500 emissions per volume. A sufficient overlap between planes is ensured by making 64 emissions using 11 defocused elements. To ensure the same focusing over the

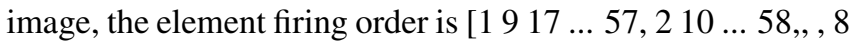
16 ... 64], so that the outer parts of the image are beamformed with received data from the full aperture.

The benefit of using this rotating aperture approach is that the point spread function is formed from all directions, and the effect of the elevation focus can be diminished to give point spread function focused in all three dimensions.

An example of such an image is shown in Fig. 11, which was scanned from a cyst phantom. Different scan planes from the acquired volume are shown.

\section{SYNTHETIC APERTURE FLOW IMAGING}

It is generally believed that SA imaging cannot be used for flow estimation based on the fact that the images are acquired over several emissions, and that they therefore cannot image moving objects. This statement is true, if the correctly summed image of the object is needed, but this is not the case. A flow imaging system uses the correlation between two measurements to derive either a phase or time shift to determine the velocity [20]. There is no demand that the image needs to be a correct depiction of the structure, which it actually is not, since the wavelength is much larger than e.g. the red blood cells. What is needed are measurements taken in exactly the same way to give signals where the only change is the motion [19]. A system that can do this is shown in Fig. 12. Here only two emissions are made for each SA. The image made by combining the emissions $[(n-3),(n-2)]$ is obtained in exactly the same way as for emissions $[(n-1),(n)]$. Corre-

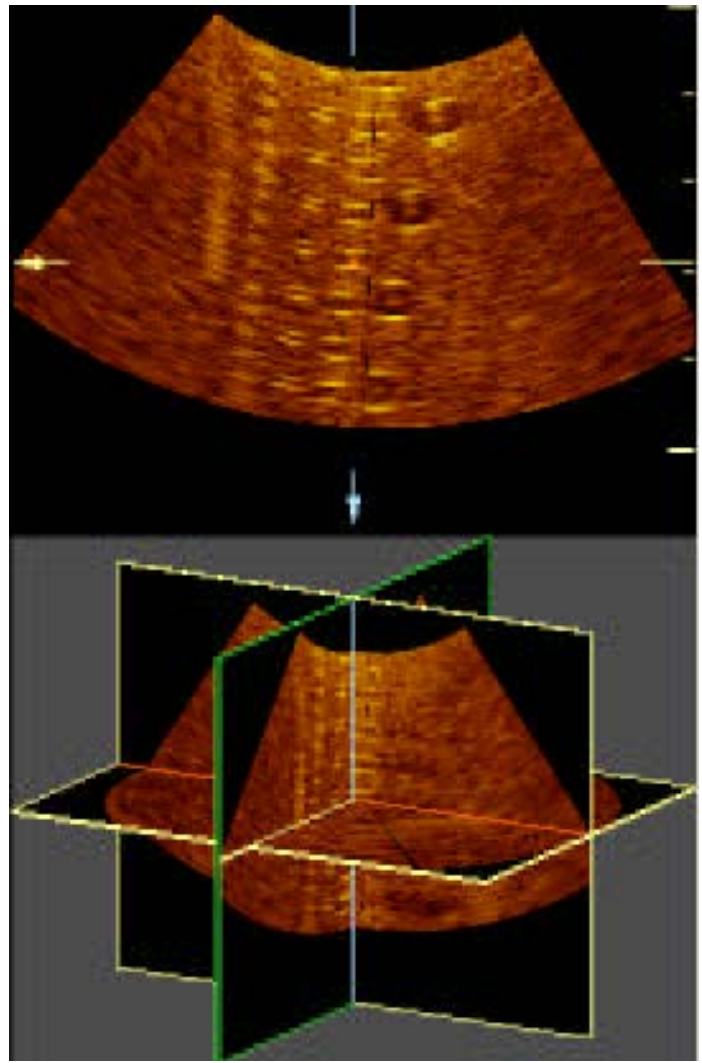

Figure 11: Three-dimensional image acquired by the rotating phased array transducer from a cyst phantom.

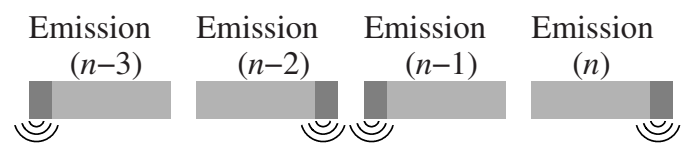

Low-resolution images
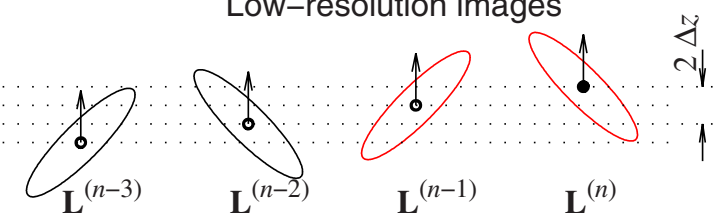

$\mathbf{L}^{(n)}$

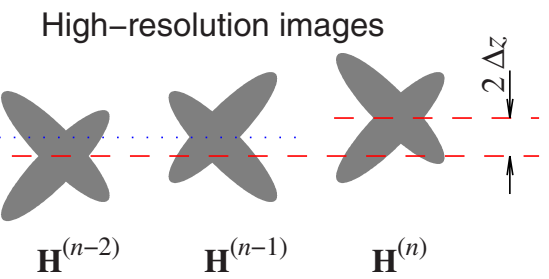

Figure 12: Creating several successive high-resolution images (from [19]). 


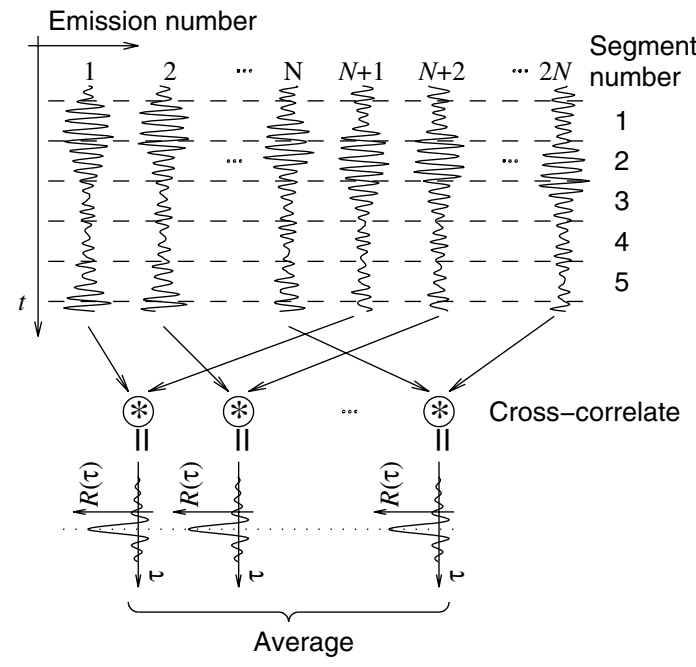

Figure 13: Creation of the cross-correlation function and its averaging over several emissions (from [19]).

lating these images and finding the shift in position can, thus, give the velocity. It should also be noted that the image pairs $[(n-2),(n-1)]$ and $[(n),(n-1)]$ also can be correlated and will give the same correlation function. A new correlation function can, thus, be made after each emission [19] and all these functions can be averaged to give a good estimate as shown in Fig. 13. The approach can be extended to any number of emissions, but it must be ensured that the correlation functions are roughly alike, so the number of emissions is limited by the acceleration of the flow.

Since the approach measures data in all direction of the image simultaneously, all data at all image points are available at all times. Much more advanced echo canceling schemes can therefore be employed using the continuous data, and the initialization problems in current filters are avoided. The estimates can also be averaged over many more emissions to increase the accuracy.

Results from using the approach are shown in Fig. 14. Flow in a circulating flow rig has been measured using the RASMUS system. A $7 \mathrm{MHz}$ linear array probe with 64 elements was used. Eleven elements were used in transmit with 4 separate transmissions of a $20 \mu$ s chirp. All 64 elements in receive were sampled. Using only 24 emissions gave the velocity profiles shown, were the solid line is the mean of the profiles and the dashed lines are \pm 3 standard deviations. The dashed-dotted line is that predicted by a mass flow meter.

The approach can also be used in-vivo. The image shown in Fig. 15 was acquired using only 24 emissions from the carotid artery of a 28 years old male. The pulse repetition frequency is $7 \mathrm{kHz}$, so more than 290 independent images can be presented per second.

A further advantage of SA imaging is that beamformation can be done in any direction. It is, thus, possible to beamform

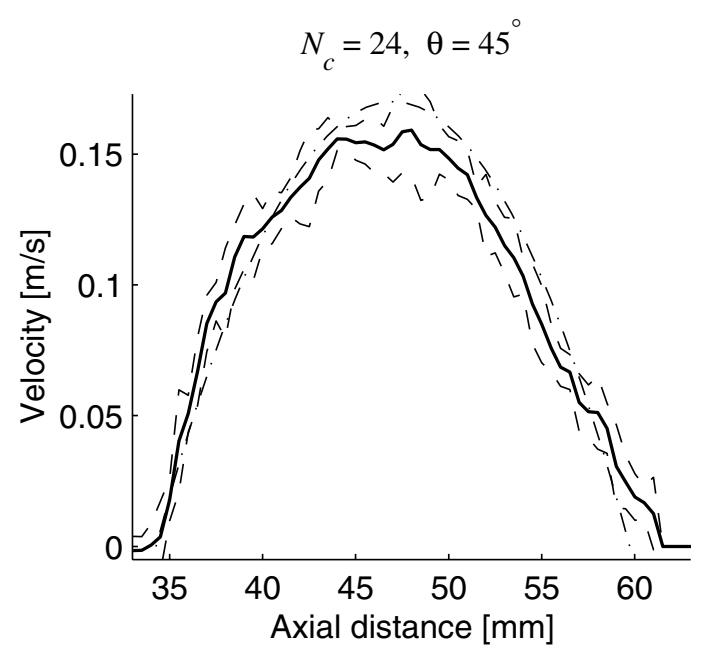

Figure 14: Velocity profile measured on a flow phantom (from [19]).

the received data along the flow lines to track the flow and thereby find the correct velocity magnitude as suggested in $[21,22]$. An example of this approach for a flow angle of 60 degrees is shown in Fig. 16 for flow rig data. The RASMUS system has been used for the data acquisition together with the $7 \mathrm{MHz}$ linear array. Eight emissions spread out over 128 elements has been made using 11 transmitting elements and a $20 \mu$ s chirp. Data from 128 elements have been sampled by using the system's multiplexers, and 128 emissions has been used for making the estimates. Data from 20 profiles is shown at the top and the mean \pm 3 standard deviations is shown on the bottom. The relative standard deviation compared to the peak velocity is $0.36 \%(0.65 \mathrm{~mm} / \mathrm{s})$. Using the same setup for the purely transverse flow a std. of $1.2 \%(2.1 \mathrm{~mm} / \mathrm{s})$ is obtained. A full color flow image can also be made from the data, and this is shown for the transverse flow in Fig. 17. The color scale indicates the velocity along the flow direction, where red hues indicate forward flow and blue reverse flow. The intensity of the color indicates the velocity magnitude.

Finally, an in-vivo image of the carotid artery of a 29 years old male is shown in Fig. 18 using the same set-up as for the flow rig experiment. Flow in both the carotid artery and the jugular vein is seen. The velocity along the flow direction is shown giving the velocity magnitude in the vessel.

\section{CONCLUSION}

It has been demonstrated that synthetic aperture imaging can be used for both anatomic and flow imaging. The method can increase both depth of penetration, focusing, and contrast by employing a group of elements in transmit along with a coded transmission. It was also demonstrated that SA imaging can be used for directional flow imaging, where data is continu- 

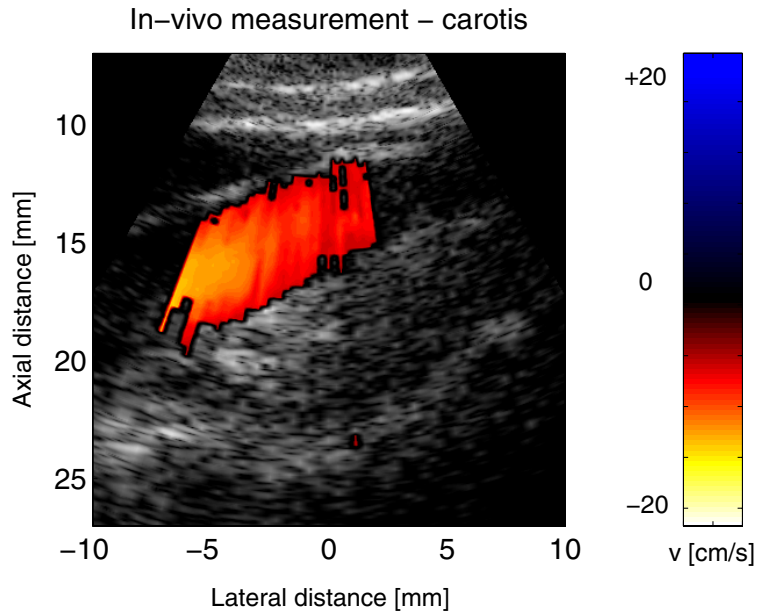

Figure 15: In-vivo synthetic aperture flow image of the carotid artery.
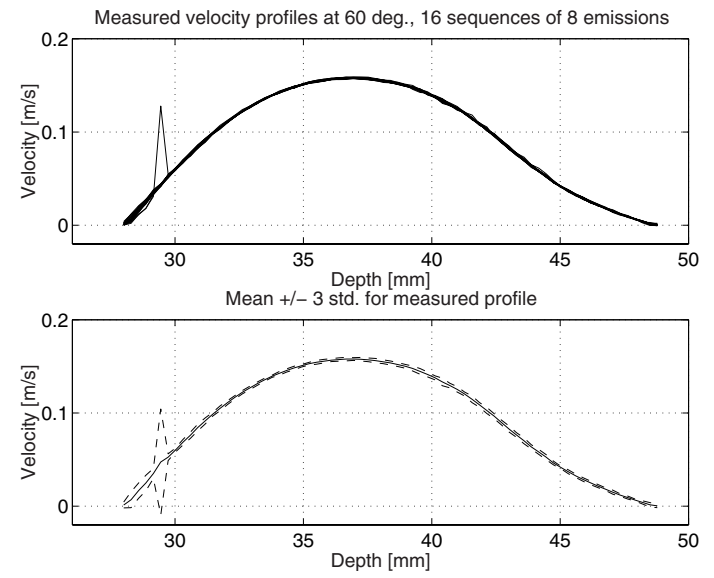

Figure 16: Estimated profiles from the flow rig at a 60 degree flow angle. The top graph shows the 20 independent profiles estimated and the bottom graph shows the mean profile (solid line) \pm 3 standard deviations (dashed lines).

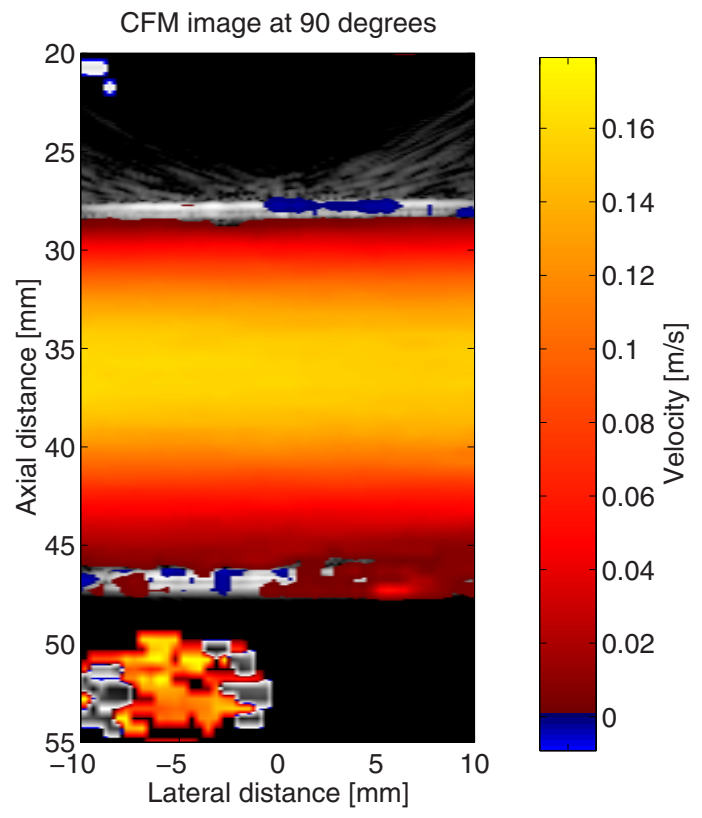

Figure 17: Color flow map SA image of flow in a flow rig at a 90 degree flow angle obtained by using directional beamforming along the flow lines. The color scale indicates the velocity along the flow direction, where red hues indicate forward flow and blue reverse flow.

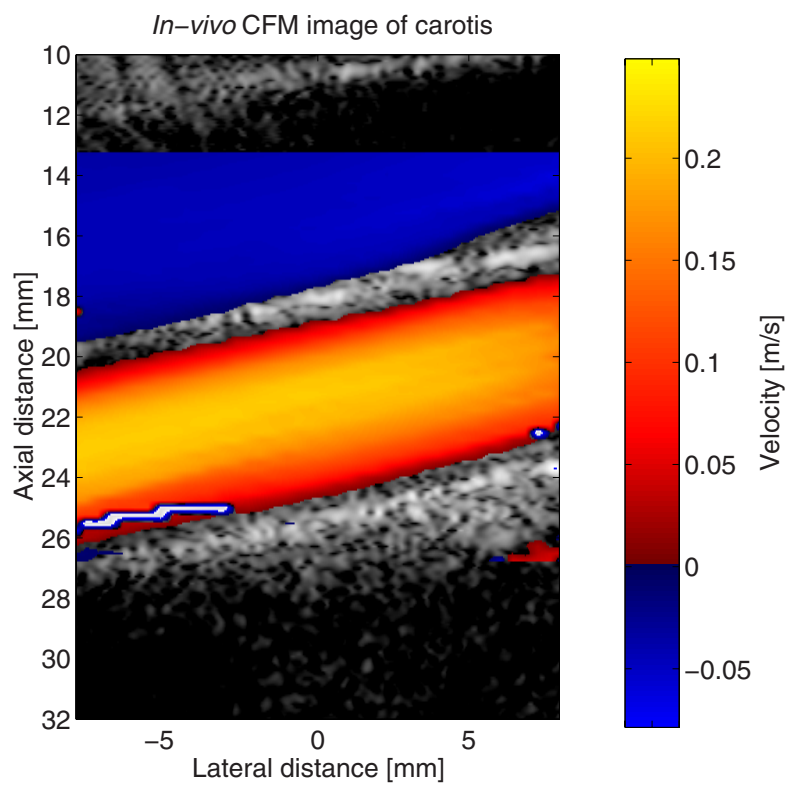

Figure 18: In-vivo color flow map image at a 77 degree flow angle for the jugular vein and carotid artery. The color scale indicates the velocity along the flow direction, where red hues indicate forward flow and blue reverse flow. 
ously available in all directions of the image. The estimates can hereby be improved, and it is possible to determine a flow transverse to the traditional ultrasound beam. The approaches can also be used to acquire data from many more elements than traditional scanners by using multiplexers and the image quality can hereby be even further increased. This can also be used for making fast and high-quality three-dimensional images.

\section{ACKNOWLEDGEMENT}

This work was supported by grant 9700883, 9700563 and 26-01-0178 from the Danish Science Foundation, by the Academy of Technical Sciences, by Frode Nygaards Foundation, and by B-K Medical A/S, Herlev, Denmark. I/O Consulting A/S, Valby Denmark made the electronic design and manufacturing of the RASMUS system.

\section{REFERENCES}

[1] D. P. Shattuck, M. D. Weinshenker, S. W. Smith, and O. T. von Ramm. Explososcan: A parallel processing technique for high speed ultrasound imaging with linear phased arrays. J. Acoust. Soc. Am., 75:1273-1282, 1984.

[2] O. T. von Ramm, S. W. Smith, and Henry G. Pavy. High-speed ultrasound volumetric imaging system Part II: Parallel processing and image display. IEEE Trans. Ultrason., Ferroelec., Freq. Contr., 38:109-115, 1991.

[3] S. Bennett, D. K. Peterson, D. Corl, and G. S. Kino. A real-time synthetic aperture digital acoustic imaging system. In P. Alais and A. F. Metherell, editors, Acoust. Imaging, volume 10, pages 669-692, 1982.

[4] K. Mayer, R. Marklein, K. J. Langenberg, and T. Kreutter. Three-dimensional imaging system based on Fourier transform synthetic aperture focusing technique. Ultrasonics, 28:241-255, 1990.

[5] L. J. Busse. Three-dimensional imaging using a frequency-domain synthetic aperture focusing technique. IEEE Trans. Ultrason., Ferroelec., Freq. Contr. 39:174-179, 1992.

[6] M. O'Donnell and L. J. Thomas. Efficient synthetic aperture imaging from a circular aperture with possible application to catheter-based imaging. IEEE Trans. Ultrason., Ferroelec., Freq. Contr., 39:366-380, 1992.

[7] J. T. Ylitalo and H. Ermert. Ultrasound synthetic aperture imaging: monostatic approach. IEEE Trans. Ultrason., Ferroelec., Freq. Contr., 41:333-339, 1994.

[8] S. I. Nikolov. Synthetic aperture tissue and flow ultrasound imaging. $\mathrm{PhD}$ thesis, Ørsted•DTU, Technical University of Denmark, 2800, Lyngby, Denmark, 2001.
[9] S. I. Nikolov, K. Gammelmark, and J. A. Jensen. Recursive ultrasound imaging. In Proc. IEEE Ultrason. Symp., volume 2, pages 1621-1625, 1999.

[10] J. A. Jensen, O. Holm, L. J. Jensen, H. Bendsen, H. M. Pedersen, K. Salomonsen, J. Hansen, and S. Nikolov. Experimental ultrasound system for real-time synthetic imaging. In Proc. IEEE Ultrason. Symp., volume 2, pages $1595-1599,1999$.

[11] J. A. Jensen. Field: A program for simulating ultrasound systems. Med. Biol. Eng. Comp., 10th NordicBaltic Conference on Biomedical Imaging, Vol. 4, Supplement 1, Part 1:351-353, 1996b.

[12] M. Karaman, P. C. Li, and M. O’Donnell. Synthetic aperture imaging for small scale systems. IEEE Trans. Ultrason., Ferroelec., Freq. Contr., 42:429-442, 1995.

[13] T. X. Misaridis and J. A. Jensen. An effective coded excitation scheme based on a predistorted FM signal and an optimized digital filter. In Proc. IEEE Ultrason. Symp., volume 2, pages 1589-1593, 1999.

[14] T. Misaridis. Ultrasound imaging using coded signals. PhD thesis, Ørsted•DTU, Technical University of Denmark, Lyngby, Denmark, 2001.

[15] K. Gammelmark. Multi-element synthetic transmit aperture imaging using temporal encoding. Master's thesis, Ørsted•DTU, Technical University of Denmark, Lyngby, Denmark, 2001.

[16] K. L. Gammelmark and J. A. Jensen. Multi-element synthetic transmit aperture imaging using temporal encoding. In Proc. SPIE: Progress in biomedical optics and imaging, volume 3, pages 25-36, 2002.

[17] G. R. Lockwood, J. R. Talman, and S. S. Brunke. Realtime 3-D ultrasound imaging using sparse synthetic aperture beamforming. IEEE Trans. Ultrason., Ferroelec., Freq. Contr., 45:980-988, 1998.

[18] A. Nguyen-Dinh, P. Mauchamp, N. Felix, R. Dufait, P. Auclair, and A. Flesch. Integrated mechanism based multiplane/3D ultrasonic imaging probes. In Proc. IEEE Ultrason. Symp., volume 2, pages 1147-1150, 2001.

[19] S. I. Nikolov and J. A. Jensen. Velocity estimation using synthetic aperture imaging. In Proc. IEEE Ultrason. Symp., pages 1409-1412, 2001.

[20] J. A. Jensen. Estimation of Blood Velocities Using Ultrasound: A Signal Processing Approach. Cambridge University Press, New York, 1996.

[21] J. A. Jensen and I. R. Lacasa. Estimation of blood velocity vectors using transverse ultrasound beam focusing and cross-correlation. In Proc. IEEE Ultrason. Symp., pages 1493-1497, 1999.

[22] J. A. Jensen. Efficient focusing scheme for transverse velocity estimation using cross-correlation. In Proc. of SPIE: Progress in biomedical optics and imaging, volume 4325, pages 242-256, 2001. 